\title{
FAULT DIAGNOSIS OF COMPUTER NUMERICAL CONTROL (CNC) MACHINE USING CLOUD KNOWLEDGE BASE AND MONITORING SYSTEM
}

\author{
Nashwa Mosaad ${ }^{1, *}$, Khaled A. Elshafey ${ }^{2}$, Ahmed N. El- Mahdy ${ }^{3}$ \\ ${ }^{1}$ Systems and Computer Engineering Department, Faculty of Engineering, Al-Azhar University, Cairo, Egypt. \\ ${ }^{2}$ Systems and Computer Eng. Dept., Faculty of Engineering, Al-Azhar University, Cairo, Egypt. \\ ${ }^{3}$ Systems and Computer Eng. Dept., Faculty of Engineering, Al-Azhar University, Cairo, Egypt. \\ *Corresponding Author E-mail: nashwamosaad.2014@Azhar.edu.eg
}

\begin{abstract}
In the world of manufacturing, numerical control machine occupies a major rôle. Recent studies on CNC machines aim to rapidly evolve CNC Controller towards smart production and flexible production lines. As an essential part of system reliability analysis, fault classification analysis is critical in the aspects of reliability design improvement. Most original Equipment manufactures do not accept the effect of machine reactions on component quality when the equipment fails. The errors coming from servo parameters for the absolute system are indicated by newly produced CNC machines. Alarm information is used only for attention, warning, and not for safeguarding work pieces against direct harm to quality. This paper aims to develop a fault diagnosis system of the CNC through monitoring the alarm panel of CNC drivers using a webcam, raspberry pi 3, Optical Character Recognition (OCR) and cloud knowledge base. By using the proposed diagnostic system, the efficiency, accuracy, speed of development, and maintenance of the CNC controllers are improved.
\end{abstract}

KEYWORDS: Fault diagnosis, Raspberry Pi 3, Machine Tool Monitoring, Optical Character Recognition (OCR), and Cloud Knowledge Base.

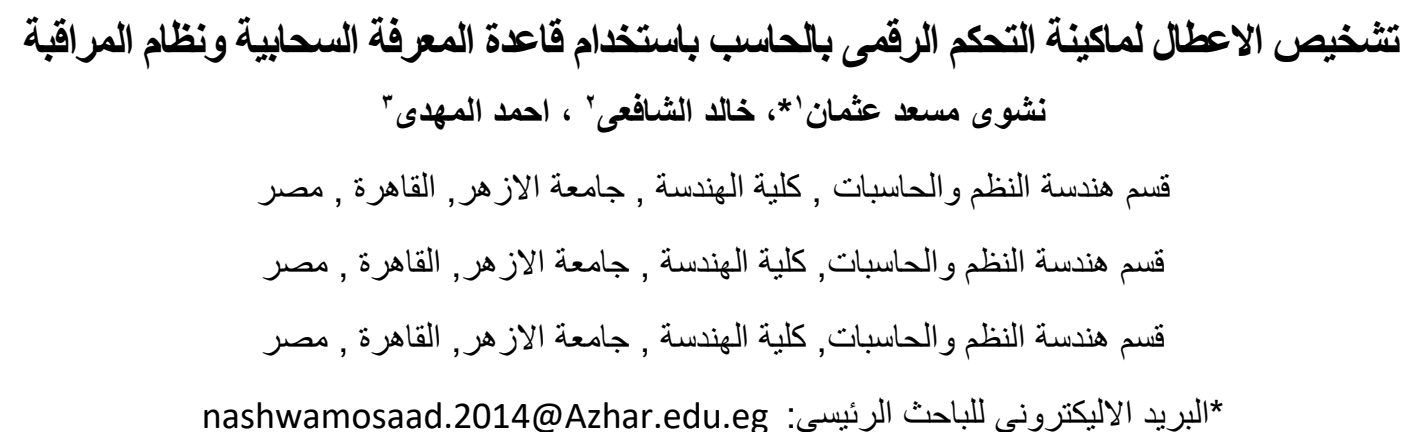




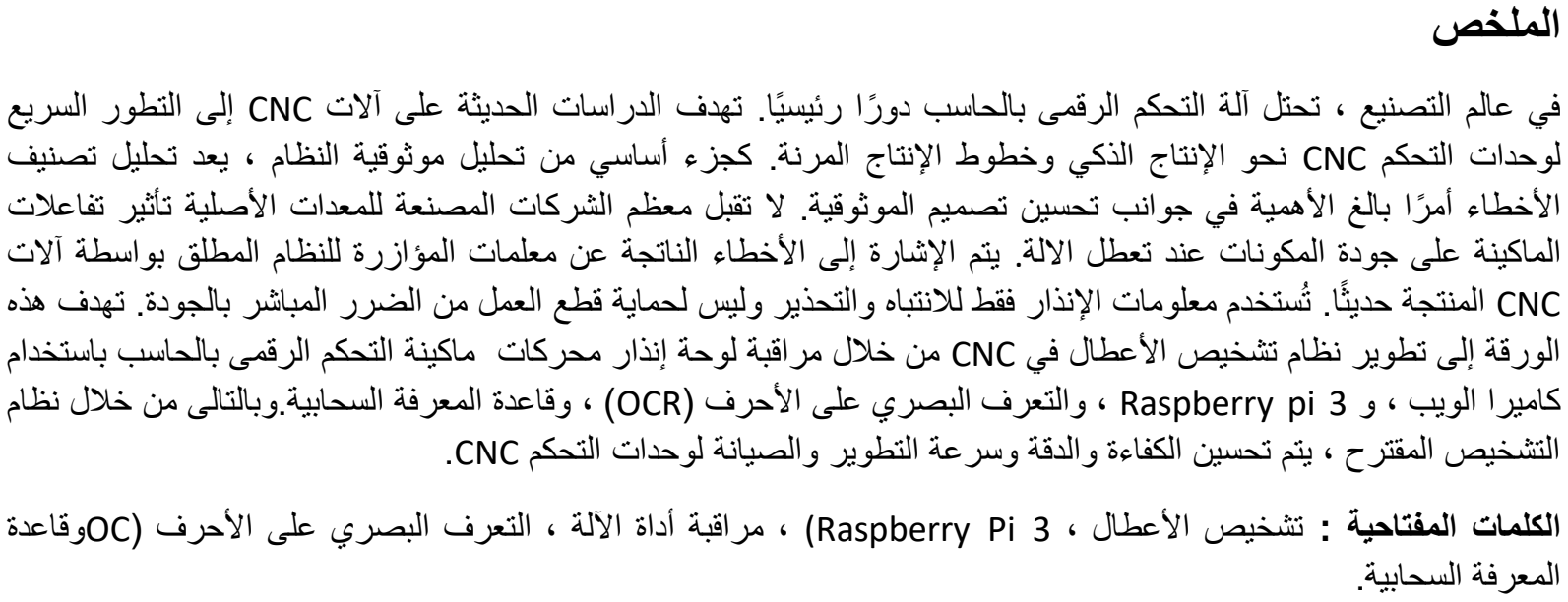

\section{INTRODUCTION}

As an essential part of system reliability analysis, fault classification analysis is particularly critical in the aspects of reliability design improvement. Most original equipment manufacturers do not accept the effect of machine reactions on component quality when the equipment fails. The main approach to solve the problem is to design preventive measures at the manufacturing level. However, the customer also discovers the need for equipment failure management at a time when the component quality issue triggered by the alarm is unexpectedly being confronted.

Some improvement methods are urgently needed to avoid quality risks like fault monitoring and fault repair. The basis of a configurable CNC system is the use of common hardware, software platforms, standard communication channels, and open communication protocols. Traditional CNC systems have the scalability of the control system depending on the specific process equipment [1]. The Internet of Things (IoT) allows us to collect and share data using the cloud knowledge base, and big data. Data support stores and analyzes the data obtained in an efficient manner and analytics provide useful knowledge for human intellectual job decision-making [2].

The distance between the control system and the machine tool is still limited by the medium which the Field bus uses. The machine tool used expects to reduce the expenditure and increase the operating rate of the machine tools. Modern control systems are not only costly, but also increasingly complex, resulting in high costs for maintenance, commissioning, and repair. Nevertheless, due to its hardware architecture, the control system's updating capacity and expandability are very limited. The supplier of machine tools expects more opportunities to provide affordable services in the repair and maintenance of their products and the upgrading of legacy systems. Cloud services in manufacturing are regarded as a promising way of organizing manufacturing tools around the world that are virtualized, distributed, and packaged as services [3, 4].

The supervision of technical processes and the quality management of systems shall be aimed at showing the present state (conditional monitoring), showing undesired or unauthorized states, and taking effective measures to prevent harm or injuries. Deviations from normal process behavior are the product of faults and errors that can occur, allotted to several causes. They can result in malfunctions or failures sooner or later [5].

At present, there are very little researches on alarm control of CNC machines, mainly focusing on the diagnosis and processing of fault alarms [6]. Some scholars study programmable logic control (PLC) Methods to ensure the safety of CNC machines and avoid errors in operation, but there is no part quality alarm design study, it is an expensive method 
and does not give an error diagnosis. To study alarm control, the alarm classification method is analyzed first [7].

Alarm classification can be divided into system alarm and OEM alarm. CNC system manufacturers such as Siemens, Fanuc, and other companies classify the fault information according to the function or structure of the system. Alarm information has nothing to do with safety or quality assurance. The alarm information is only used for reminding, and will not bring direct damage to the quality. Alarm status during the alarm, the CNC machine tool can have five action states: "alarm prompt" "pause" "emergency stop" "fault stop" and "abnormal stop" There is no design specification for the alarm.

"Alarm prompt" means that the operating panel shows alarm information, but the state of the system is not affected and still regular. "Stop" means the rotation of the machine tool axis, including the machine coordinate axis, the spindle, and some auxiliary axes.

"Pause" means the machine tool axis movement pause, including the machine coordinate axis, the spindle and some auxiliary axes.

"Emergency stop" is an alarm generated when the machine tool is in an emergency. All the axes immediately stop and all the power to drive devices is cut off.

"Fault stop" is the stop caused by equipment failure, which may be controlled by a CNC system, relay, or drive module.

"Abnormal stop" refers to the sudden machine action stop due to the failure of a machine tool or external reasons. At this time, the CNC system is out of control and unable to control the mechanical system.

Machine tool failure primarily affects accuracy when the alarm affects the parts; it must first be in the state that the alarm occurs during the machining process. At this time, the tool is cutting the parts or the spindle deviates abnormally due to fault may damage the parts.

If the "pause" state is activated, the sudden change in the cutting process may form a trace that is normally less than $0.02 \mathrm{~mm}$, which only has a slight impact on the surface quality. However, if the relay or servo drive is used to enable the "pause," some broad marks can be created largely due to the inertia or the axis brake. So there is a quality risk. Some alarm information must be cleared through machine restart or driver reset.

The machine response is controlled by the system and hardware components. It is slower than an emergency stop and has a greater impact on the part quality. When an abnormal stop appears, the coordinate axis may not be able to break, so the abnormal axis movement could reach several millimeters due to inertia. The gravity axis without balance pressure may even fall to the lowest due to gravity, resulting in parts scrapping and machine damage. In highprecision machining such as boring, shape accuracy may be affected and surface pattern or steps may appear. Emergency stop has a significant effect on the machining that the coordinate axes shift abnormally due to inertia or brake delay, resulting in a problem with accuracy. An abnormal stop is most likely to result in distortion of work piece, which must be avoided with all efforts [8].

\section{RELATED WORK}

There are a lot of studies on hypotheses, processes, and technology for fault diagnosis. $\mathbf{H e} \mathbf{X}$ (2016) [9] discussed the reliabilities of typical subsystems of NC machine tools. Besides, a range of key issues and challenges remain to be addressed in the reliability study of NC machine tools, and opportunities for further research are found. Model, not just the life and reliability of the system can be analyzed, device failure mechanism, effects internal properties, external variables, and device parameters; it can also be observed. Default unit-agent model, device level the failure agent model, the hierarchical model structure, and the simulation control method have been defined separately. An illustration of the machine tool feed system 
model was given. The author focused on the cutting tool failure due to wear frequently of the spindle. He considered these parts as the most important factor in the quality of production. However, the spindle moves through the driver and thus controlling through Prevent excessive friction by stopping the spindle motor when the torque is increased to prevent wear is the best. Moreover, a reliability estimation approach to the cutting tools based on a logistic regression model by using vibration signals had been proposed not optimal with the CNC high precision machines.

Goyal D (2015) [10] presents the analysis of the research presented by different researchers on Instruments used for vibration analysis and signal processing techniques for condition monitoring Machines produce vibrations during the procedure that cause machine tools to deteriorate. It inevitably causes certain subsystems or the computer itself to malfunction analyzing the vibration signatures. The type and extent of any damage to machines and components or any damage to components may be used to detect. The machine-related maintenance decisions Condition-based tracking has become an underlying condition. It's an important technique for ensuring the accessibility of the machine by timely maintenance activities and reducing and Maintenance for a breakdown. It's a study of vibration monitoring methods and signal processing techniques, including vibration measurement methods and signal processing techniques, which were performed for machines and machine tools. The disadvantage of fault diagnosis depending on vibration and signal processing techniques is the noise and needing to high perform acting filters. It is a hard job to extract the feature from the acquired signal as interferences are occurred due to the presence of noise.

Siddhpura A. (2013) [11] Focusing on the spindle and feed drive fitness Optimization of cutting tool operation life minimizes loss of life. Failure of the instrument and, subsequently, economic losses due to downtime Risks of either early tool replacement or cutting and scrap it. In most instances, the TCM Intelligent Tool is combined with Condition-based maintenance $(\mathrm{CBM})$, and/or predictive maintenance $(\mathrm{PdM})$ is preferred over preventive maintenance for fixed maintenance Predetermined time intervals based on historical health tool data. The disadvantage of this method it's could increase the amount of costly unnecessary Maintenance. Since chipping and breaking tools are sudden and random in nature, most of the TCM systems concentrate on the observation of gradual tool wear.

Li, D. H., (2016) [12] focuses on the operation of radial base function (RBF) networks detection and classification of faults in the motorized spindle. As well as a high-performance data-driven model, RBF networks advantage to solve nonlinear problems and dealing with nonlinear problems conflicting samples in the training phase. In this area of study, reporting, including rotational speed, temperature, and acceleration Signals with three axes

$(\mathrm{X}, \mathrm{Y}$, and $\mathrm{Z}$ ) are obtained from the dynamic balancing platform for evaluation of working conditions and to detect the possible faults of the motorized spindle. This paper only injected the failure with damage on the bearings, not focused on increasing the types of injected failures to check whether RBF networks can recognize failures from several types of faults.

Qiang Zhou (2017) [13] A Knowledge Modeling Framework for Default Diagnosis of Machine Tools Based on Formal Semantics(KMM-MTFD) is proposed to construct a free, shared, and scalable Ontology-based Default Diagnosis Knowledge Model for various machine tools (OKM-MTFD). First, the proposed predicate-logic-based fault-element analysis approach is used to analyze the fault diagnostic domain and to extract common domain information, which allows the development of the core ontology of OKM-MTFD to ensure formal semantics. Next, using the proposed two-stage classification method for fault elements and external ontology reference methods, the core ontology can be applied to the OKMMTFD form for machine tools.

The predicate-logic-based method of analysis of the fault elements is used to evaluate the linguistic components of the fault elements to obtain a collection of standard terms for the fault diagnosis of machine tools, maintaining unified formal semantics. By adopting a 
predicate logic, the approach can also solve the problem of expression diversity that exists in current research and thus improve semantics in the fault element description. Also, any linguistic component is semantic and can be associated with others by appropriate predicates to help the development of new information that can be edited, reused, and retained under practical needs, providing basic elements and logical support.

The predicate-logic analysis method of the fault elements is performed as FP, FM, FC, and FL should be separated from the original fault diagnostic records. Second, the properties of each fault aspect should be extracted in the form "Subject-Verb-Object' in which the subject and the object represent the body or property and the verb represents their relationship. Third, each property formatted in "Subject-Verb-Object' can be viewed as an atomic proposition and can be further divided into individual terms, predicates, quantifiers, etc. For all properties and fault elements, the process should be carried out. Finally, after reviewing a vast number of fault reports, libraries of individual words, predicates, and quantifiers are obtained. Consequently, these libraries are analyzed and summarized to build a library of terminology for machine tool fault diagnostics. Individual terms, as subject or object, can be refined to classes in ontology, such as fault element classes and fault element attribute classes; predicates can be refined to object properties in ontology; quantifiers. For the analysis of massive failure diagnostic records, rough set theory can be used for the analysis and extraction of fault elements to construct- ontology. The disadvantages of this method are the model is not able to accurately discriminate the cutting operations; this could be since some aspects of the conflicted operation.

Alex Patricio (2020) [14] The article presents the design and implementation of an architecture based on cyber-physical systems (CPS), which are structures controlled and monitored by computer algorithms and closely integrated with the internet, to track variables acquired from a computer numerical control (CNC) machine and a robot manipulator. This architecture uses specific components and is completely implemented on the raspberry pi. Also, the architecture uses a multi-client-server topology designed in the python language for effective global visualization of the variables acquired from the connected devices. Human User Interface (HMI) has also been developed for the real-time visualization of key system indicators selected according to user requirements. Besides, the architecture was checked in the process of industrial component machining and the stability of the data acquisition method was validated based on knowledge of the machine state. The method provided often becomes a valuable tool in the decision-making process, as it uses constantly updated information, statistics, and the trend history of each machine that makes up the CPS. The disadvantages of this architecture are:

HMI needed to be developed for real-time visualization; it doesn't prevent losses in materials, the author focused on the main CNC monitor who just shows the operating system error message and neglected the alarm display panel monitoring which shows codes that represent any error of the machine. It is considered expensive because of using a robotic arm and smart monitors.

\section{PROBLEM FORMULATION}

A torque-based engine consisting of a rotary actuator or linear actuator is a servo motor. This type of motor makes it possible to precisely change the angular and linear direction, torque, velocity, and acceleration controls. Modern servo systems contain four components: motor, drive, controller, and feedback unit. Usually, servo motors are very small, but they still pack a lot of power and performance, which is why they are used for many applications, including industrial ones. In industries such as robotics, metal cutting and shaping, printing, woodworking, conveyor belt setups, and more, you'll find them.

The inertia load should never exceed ten times its capacity when choosing a servo motor. There are several distinct kinds of servo motors and drives, but all are a means to an end. 
They eventually power machinery and machines of industrial grade and keep a plant completely operational. However, they can fail, like everything.

It can be expensive for your business and crew when that happens. Reparations can take longer when you don't have an experienced repair team, keeping the equipment down for hours, days, or even weeks.

Troubleshooting of servo drives and troubleshooting of servo motors are the lifeblood of problem detection and fixing. The ultimate purpose is to work with the servo and occasionally run it to deduce what is going wrong. Fortunately, most of the servo drive and engine systems are connected to a warning that will sound appear on the alarm display panel when something is up as shown in figure1.

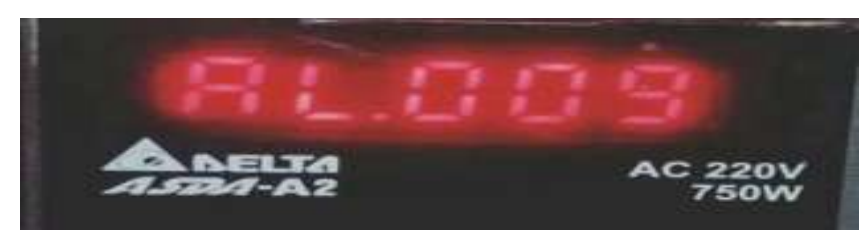

Figure1: Alarm display panel.

In this article, we will use ASDA-A2 series servo motor drivers as a case study to diagnose 555 faults as described in ASDA-A2 series servo motor drivers manual user. Servo drive troubleshooting can be a complex and hazardous operation. Working knowledge of electromechanically operated engines and actuators is needed. If the machine operator appears unfamiliar with all of these elements, outsource assistance. You'll want to be prepared before taking action as you read up on how to fix the servo motor and drive mechanism. Productivity and performance of the manufacturing process, key issues in an involved and active manner. Sustainable operation of the shop floor is planned to ensure higher machine availability, minimize idle/downtime, and achieve higher yields by constantly monitoring the shop floor environment. In general, it is traditional factories have restricted visibility and contact on the shop floor between machines and operators. If an operator is running several machine tools on the floor of the store, problems that cause shutdowns or stop machines, it cannot be detected until it appears in front of the engine drivers of the machines. He's just seeing the warning code so he stops or pause the machine tools until the problem has been corrected by the technician or maintenance responsible. Therefore there are energy and time consumption, the wasted time decreases productivity and maximizes unproductive idle. The operator task in this situation is Restricted to the monitoring of just a few machines.

Remote monitoring and cloud computing can reduce unnecessary time, energy consumption, the time for fault detection and maintenance. The monitoring system in real-time is very vital, and there are many surveillance systems available in the market. But these IP-based security systems are very costly, and the cheaper ones only stream data locally. So in this article, we built useful yet straightforward fault diagnoses and monitoring System using raspberry pi3 figure 2 , and webcam.

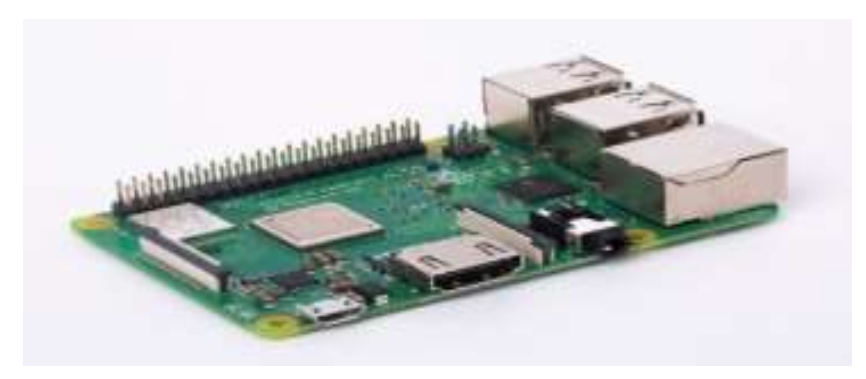


Figure 2: Raspberry Pi computer model B+.

\section{THE ARCHITECTURE OF FAULT DIAGNOSIS PROPOSED APPROACH}

The proposed approach consists of three main components:

1. Data collecting from the captured image using Optical Character Recognition (OCR) and Tesseract algorithm to detect the alarm occurring and the alarm code as shown in figure 3 .

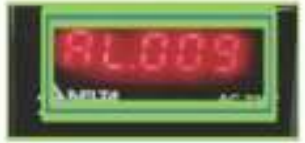

Captured inage.

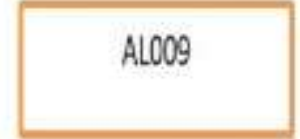

OCR Result.

Figure 3: Alarm detection.

2. Sending data to the local host PhpmyAdmin figure 4 using Apache server and PHP MySQL database for local hosting and to get the Fault diagnosis. Due to the benefits of free software, dynamism, and practicality, the Web application is developed with a PHP programming language. This language is capable of communicating with the world of the internet, fully enhancing websites that have static pages. Additionally, PHP does not create redundant copies of data, which reduces the amount of information stored on the database and avoids them becoming corrupted.

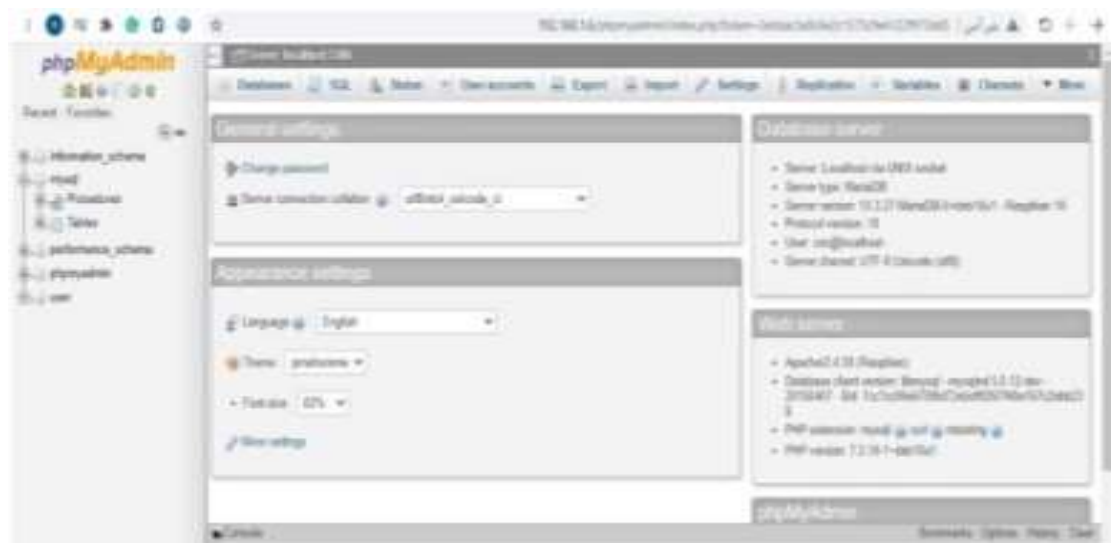

Figure 4: PhpmyAdmin.

The PHP code runs on the server and the results are sent to the browser, which shows the rendered page without wasting the resources of the machine. PHP can be used together with Hypertext Markup Language (HTML) code for Web page development and Apache, an opensource and free Hypertext Transfer Protocol (HTTP) multiplatform server. You can use the web page to query the data stored in the online database via smartphones or PCs. Whereas Ethernet standard is the solution most widely used in priority systems for interconnectivity of networks, costs of monitoring systems for micro-network Wi-Fi with free software tend to reduce; additionally, installation practicability of wireless applications is higher. The sampling time delay for viewing on the server is almost zero, so the system can be considered a realtime application .

From router to Wi-Fi devices are encrypted. Only the indented receiver can process and read data from the signal and protect the communication channel. Log monitoring is viewed from 
the local host PhpmyAdmin so you need to put your data in MySQL organized database figure 5 .
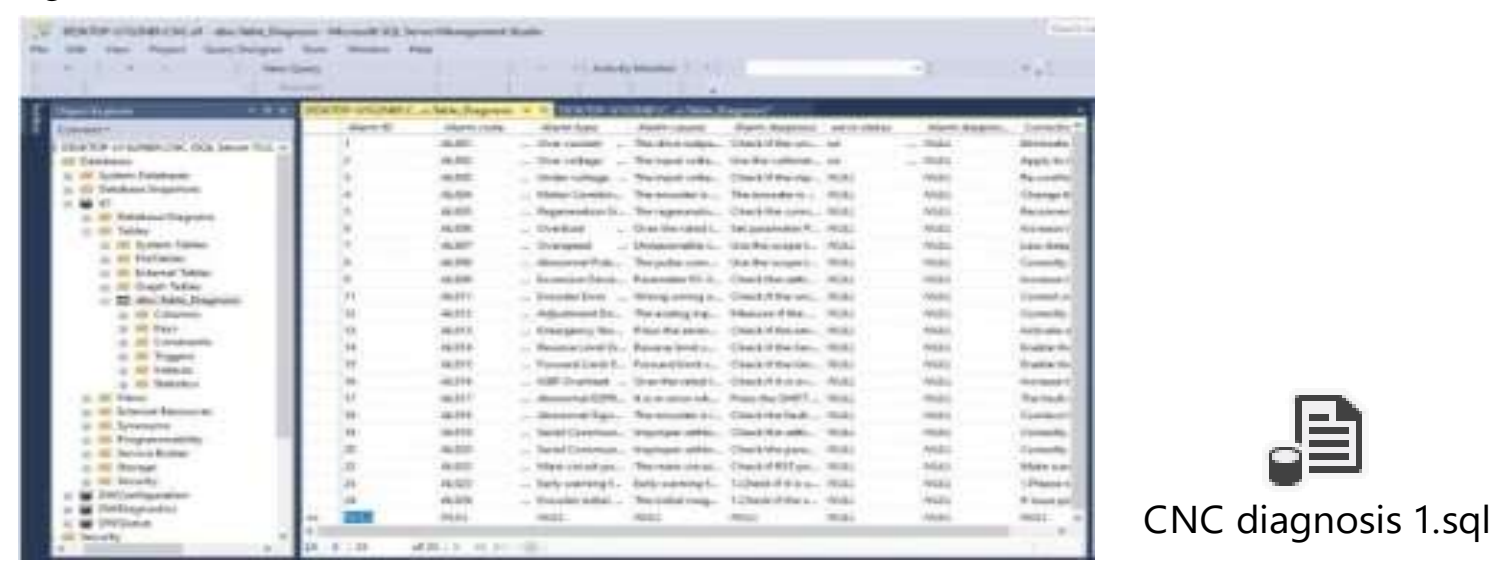

Figure 5: My SQL database design.

3. Transmission of data to the cloud server to add the alarm occurs and processing data in real-time according to user requirements .

Configuration through a communication interface provides significant advantages over sending configuration and data inserts. The developed web monitor for sending files to the server is very secure. There are two fundamental options for ensuring communication between a cloud server and client: pull and push. The pull option is implemented via a file read/write mechanism on the server. In this scenario, the system will periodically check if changes have been made to the index stored in the cloud web server register. If yes, the requested command is executed, otherwise, queries continue until there are changes.

The disadvantage of pull communication is that the user has to send several requests to the server to know if there is some change to the server, to know if there is any update of the information (pulling); this methodology increases the processing of the user's device and generates discomfort. The alternative is to invert the functions, making the server function as a client whenever a new message arrives. This model is known as push, a client-server communication model in which the server initiates communication and transfers data to a client without directly transmitting [15].

\subsection{Monitoring of Machine Drivers}

For digital image capture in computer vision, Charge-Coupled Device (CCD) and Complementary Metal Oxide Semiconductor (CMOS) sensors have been developed for several years. Recently, CMOS vision modules have been commonly used for consumer devices such as mobile phones, laptops, tablets, and digital cameras. In the manufacturing sector, machine vision has become an important method for the accurate identification of a wide variety of workpieces in the flow of products through production processes. Innovative machine learning and deep learning processes have recently improved recognition rates and coverage. With low prices and increasing the average performance of vision devices, the use of a consumer-grade webcam is a cost-saving and affordable solution to the machine vision system. For example, many studies used webcams for facial recognition, motion detection, and in-home automation systems [16, 17].

\subsection{Fault Detection Using a Webcam and Optical Character Recognition (OCR)}


To collect the data from of alarm display panel, optical character recognition (OCR) is one of the applicable technologies. Optical character recognition is an algorithm that acts as a human ability to read. After the image has been collected from a vision source, the image processing functions adjust the image to be appropriate for the identification of the character data. The OCR algorithm then determines the original expressions based on the trained data of the identifiable characters [18].

In this article, Tesseract is chosen as an OCR algorithm, Google responsible for the maintenance of Tesseract, so it is regarded as one of the most accurate OCR free and opensource engines currently available. One of the best alternative open-source methods for the development of image processing and computer vision algorithms is followed to boost the results of the Tesseract engine, gray scale, and binarization using the OpenCV (Open-source Computer Vision Library). The combination of webcam and OCR replaces the ability of the operators to read. Operational data can be obtained with sufficient resolution of the webcam.

The server device can extract useful information to the users, with supervision we could be able to prevent malfunction, follow up the driver status, errors, fault detection, and diagnosis.

The monitoring system framework consists of two modules: the client and the server. The client is responsible for storing and transmitting data from the collected HMI images of the machine tool, and the server stores the tracked data and processes events of the driver's status.

\subsection{Region of Interest:}

With the region of interest (ROI) data registered through the ROI manager, the client device manages all data recognition and webcam connections by running processes iteratively, The client has two manager ROI and OCR engines sub modules. ROI manager: responsible for character analysis, target item ROIs, based on the captured image, need to be specified, including alarm code, sort, and parameter for image processing.

ROIs were developed to facilitate intuitive and easy-to-use registration to define the appropriate input parameters. To improve user experience, upper and lower ROI limits may also be defined in this capture phase. The name of the operating data is selected in the listmenu and the data type is set automatically based on the predefined conditions. Then, the ROI manager transforms the captured image subsets by pre-processing, cropping as shown in figure 6 , and image modifications, and passes them to the OCR engine.

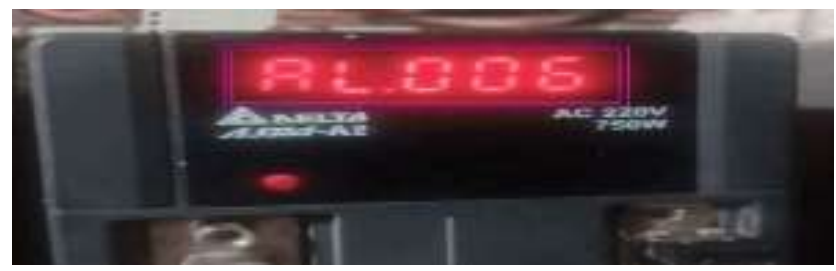

Figure 6: Region of interest.

All ROI information can be stored in a data file and the file can be loaded for the same monitoring condition in future use.

OCR engine: the OCR engine analyzes the captured image subsets using the OCR algorithm. 
Results of the OCR process have been converted based on ROI data. Due to the imperfections of the OCR procedure, some irregular results values are substituted as the previous one. This method is a naive but successful solution since most abnormal values will not be expected to change dramatically during the sample duration due to the dynamic properties of mechanical parts, such as inertia and friction, in manufacturing equipment. With the integration of the ROI manager, OCR engine, and the client on a small computer with a linked webcam, the client configuration acts as a smart sensor on the IoT network. To help the user understand the monitoring system, the ROI and OCR results are displayed in real-time on the monitor image. Wi-Fi and MQTT (Message Queuing Telemetry Transport) brokers are used for network communication to upload controlled data to the server. Server-side operations process and interpret data $[19,20]$.

\subsection{Cloud Server:}

The server architecture is specified for the processing of real-time monitoring data from the client. The design is made up of software modules; communication and data platform: to enable structured communication and data collection from the smart monitoring system as a sensor on IoT networks, the firebase platform is used as a data platform on the server. Firebase is an IoT server platform that complies with the oneM2M standards [21].

It provides all functions for IoT devices, including registration, data and repository management, system management, protection, communication and distribution management, discovery, subscription, and notification. To interconnect firebase and the end-point units, like raspberry pi and Node-js program offers MQTT bindings via a wireless network. MQTT is a simple message protocol designed for restricted low-bandwidth applications. This is also the perfect solution for applications on the Internet of Things. MQTT allows you to send commands to monitor performance, read and publish data from sensor nodes, and more.

Firebase is NOSQL using JSON (JavaScript Object Notation) is a lightweight datainterchange format. It's easy for people to read and write. It's easy for machines to search and produce. Used as the primary development platform and the Database Management System (DMBS) for data storage real-time figure 7.

Concerning implementation, the server is designed to run as either cloud computing or fog computing. With the approach of conventional cloud-based computing, the server can be placed on remote servers belonging to Google, Microsoft, Amazon, and the business. However, the responsibility for uploading data to remote cloud data centers rests with IoTrestricted resources in connected devices. The processing of data on in-field devices could make the platform scalable by reducing the time of remote processing, with low data transmission and storage. With these problems of real-time data, IoT data processing activities are moving closer to IoT data sources or data sinks. 


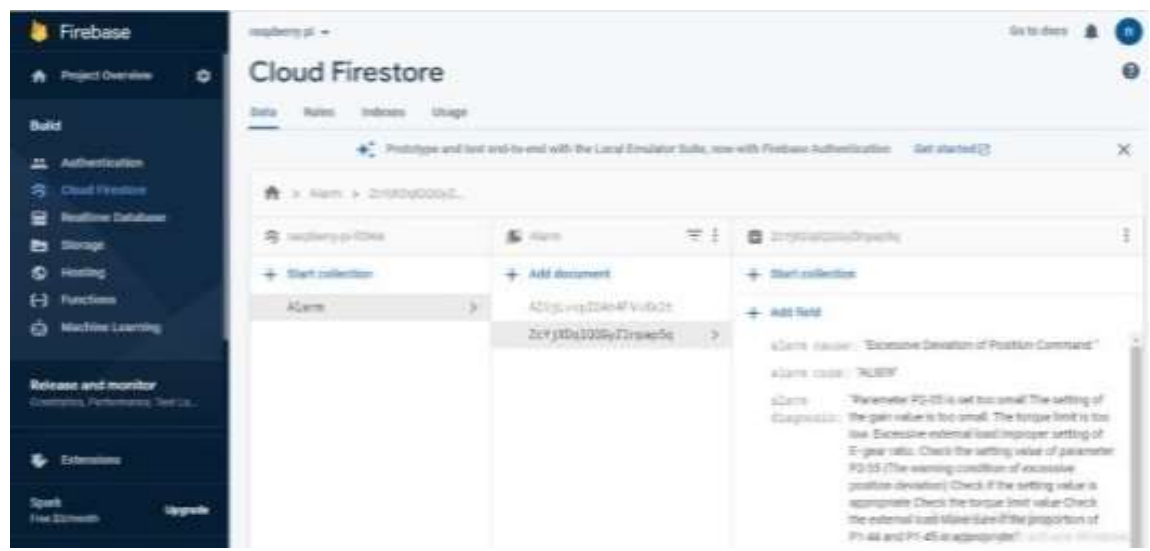

Figure 7: Cloud firebase.

Fog computing is a highly virtualized platform that provides computing, storage, and networking services between end devices (things) and conventional computer networks [22]. Thanks to the functionalities of firebase that support the smart gateway approach and MQTT broker technologies, the server can be run, send a message to smartphone or email, eliminating the need for monitoring and also maintaining a secure supply. Many popular M2M protocols like Message Queuing Telemetry Transport (MQTT) [23], Constrained Application Protocol (CoAP) [24] and Open Mobile Alliance Light Weight M2M (OWA LWM2M) are used .

There are many protocols used for IoT applications that have been compared [25][26] concluding that although each protocol has its own merits and suits different communications, however, MQTT protocol is better suited for event-based applications. MQTT provides different modes of delivery, low power consumption, and security. MQTT is a client serverbased notification protocol model. It was initially designed for Supervisory Control and Data Acquisition (SCADA) systems for oil pipelines and satellite links [27] and was later commercially developed by IBM [28]. MQTT was designed for messaging over TCP with low-bandwidth, high latency. A real-world use includes the Facebook messenger application which was based on this protocol to suit battery-powered mobile devices [29].

\subsection{Cloud Firebase Web Services}

Raspberry pi uses python language instructions to communicate to firebase IoT and Google logging web service by Oath protocol. Internet of things allows data sync from devices, sensors, and computers. Google firebase IoT provides a software development kit to easily connect hardware devices or mobile applications. IoT Device SDK enables devices to connect, authenticate, and sync messages with IoT using MQTT (MQ Telemetry Transport) standard publish-subscribe protocol Firebase SNS (simple notification to send) is a fast, formable, fully managed messaging service. It is a cloud-based mobile app notification service that sends push alerts, texts, and SMS messages: or as an enterprise messaging infrastructure. Google APIs (application interfaces) use the open authorization Oath 2.0 protocol for authentication and authorization. Google supports the Oath 2.0 protocol for those for a web server, installed, and client-side application. It can log the intrusion data in the spreadsheet and having some security credentials, which maintains real-time updates.

The IoT is the compilation of data through an embedded system. The computer uploads information to the network. Data and industrial automation for IoT and industrial automation 
service security, trust, data honesty, scalability of automation domain information privacy and interoperability constrain, limits. This paper combines the concept of raspberry Pi with the IoT, manufacturing workstations, and industrial automation use the machine uses the raspberry pi as the controller, and the server programming is implemented in the python language. The webpage is constructed as an HTML, JQuery, and Flask platform HTML template rendering for python .This system will detect the presence of an alarm quickly alert the users by sending an e-mail to their phones and computers.

Raspberry $\mathrm{Pi}$ is the most suitable microcontroller for IoT projects as it has inbuilt Wi-Fi to connect with the internet easily when the alarm signal coming from $\mathrm{CNC}$ to raspberry pi the webcam automatically takes a capture and at the same time all CNC machine motors and the G-code stop running. At the same time, the user will receive a message sent by raspberry pi through an email, that there is a malfunction in the machine, with a picture of the alarm code attached. We built a locale host containing the alarm codes database and a page to control $\mathrm{CNC}$ remotely (ON, OFF, and RESET drivers). Through the page, the user can make a query and a diagnostic message by the cause of the error and the corrective actions will be received. A cloud firebase in real-time is used to send a notification to the person who is responsible for machine maintenance as shown in figure 8 .

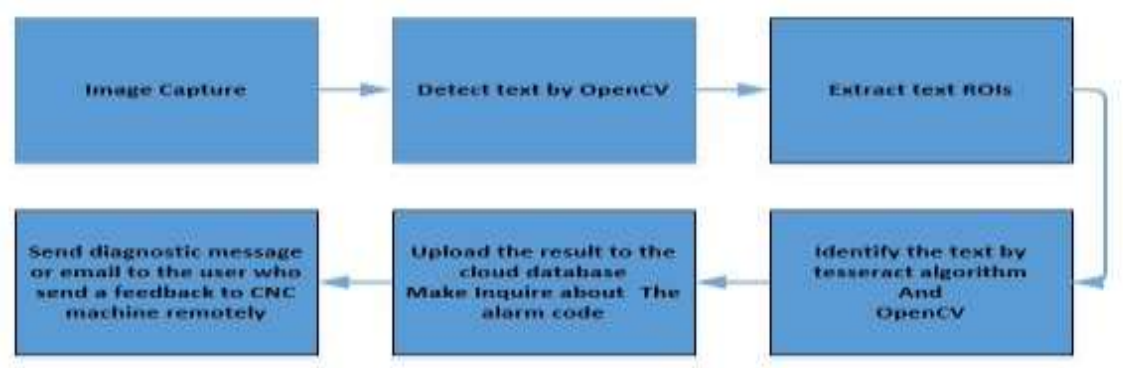

Figure 8: Working procedure.

\section{IMPLEMENTATION}

Raspberry pi board is a miniaturized fascinated computer having ample processing speed and size not bigger than a credit card. Incredible things can be done by using it. The most common updated version of the $\mathrm{Pi}$ is Model $\mathrm{B}+$, with an improved version with accessibility. Firstly, to work with raspberry pi, we need a list of things to get the desired operation and functioning figure 9.An Ethernet or Wi-Fi Adapter.

1. SD card $16 \mathrm{~GB}$ as a minimum.

2. Monitor.

3. HDMI to VGA converter but if we have LCD TV connect with HDMI cable it can be used.

4. Keyboard and Mouse. 


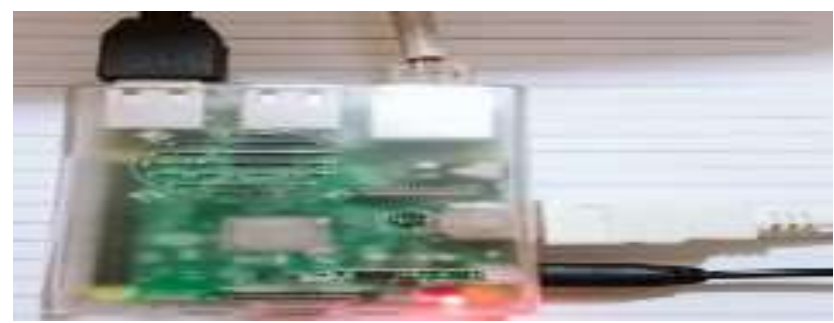

Figure 9: Raspberry pi setup.

\subsection{Raspbian Operating System}

Set up the Raspbian operating system, Raspbian image (about 2 GB) must be written to the SD card (minimum $16 \mathrm{~GB}$ ), use another computer and then put the card in the raspberry pi. The SD card functions like raspberry pi hard drive. Extra space can be used for the storage of data if a large amount of data and applications are needed.

\subsection{Raspberry pi Configuration}

In the first configuration of raspberry pi enable SSH Source Shield server figure10 this will enable secure shell access, which means that we can control your raspberry pi from a remote computer or mobile phone.

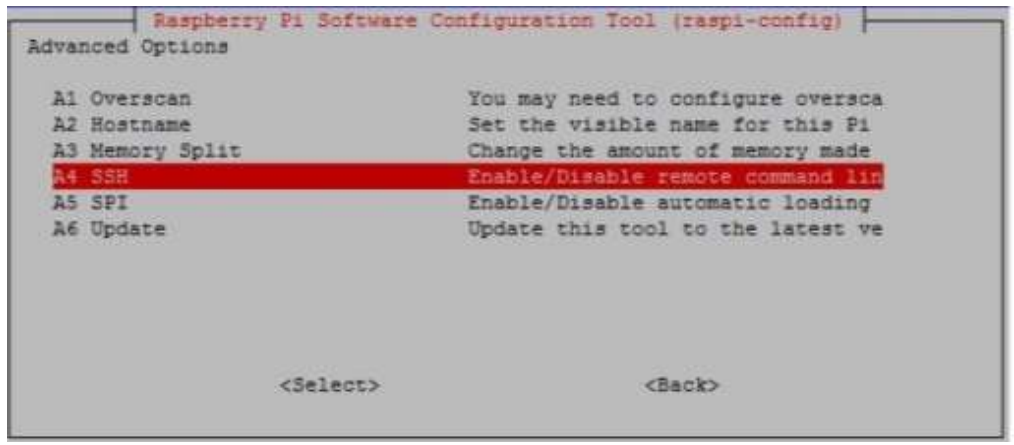

Figure 10: SSH enable.

If you are running an operating system with a graphical user interface (GUI) on your raspberry pi, open a terminal window on the computer and follow the instructions in that window. Otherwise, if you need a remote terminal, such as Putty, fig. 11 to connect to your computer, open a remote terminal on your device write the raspberry pi IP address and uses it.

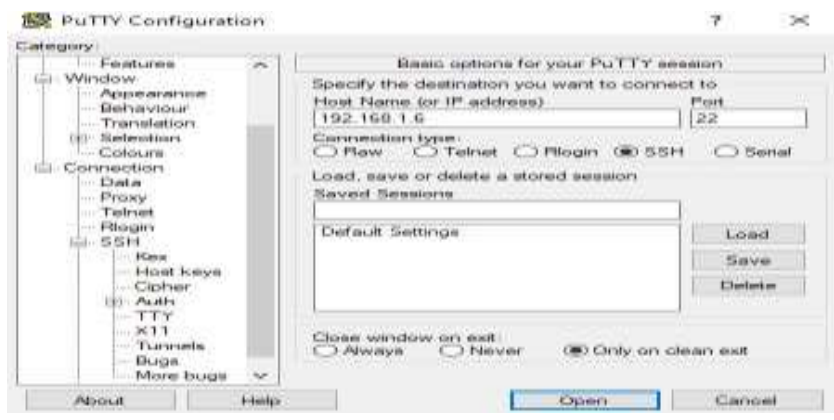

Figure 11: Putty.

Open the command line, type in:

sudo apt-get update

sudo apt-get upgrade 
You'll be given a lot of gobbledygook by each of these orders. You'll be waiting for a while. This package lists updates from different repositories and updates them to get details about the newest package releases and their dependencies.

sudo reboot

VNC protocol Virtual Network Computing and open RealVNC are needed to show raspberry pi desktop on another computer.

To install a program on Raspbian open the pi terminal and type:

sudo apt-get update

sudo apt-get upgrade

sudo apt-get install program-name -y

\subsection{The Required Libraries and Programs Installation}

Install a webcam program like fswebcam and MPlayer, for capturing the images and real-time monitoring videos. Give the raspberry pi static IP because it is needed to access raspberry pi. Download the required program and enable communication protocols from raspberry pi configuration like (i2C, SPI, UART, 1Wire) to connect with another device.

Download and install electronic control RPI. gpio GIOP (general purpose input-output) library, node js, python, WebIOPi for the internet of things and device monitor page, WebIOPi support client-server programming. Install OpenCV open computer vision, Tesseract-OCR, pytesseract for text /image recognition figure 12.

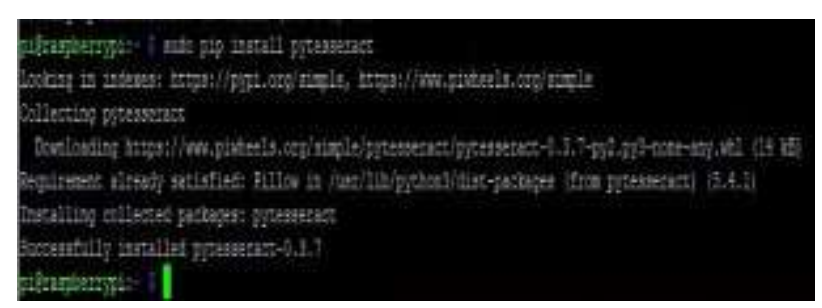

Figure 12: Pytesseract installations.

Install Apache server, PHP, and PHP-MYSQL to set up your local host figure 13.

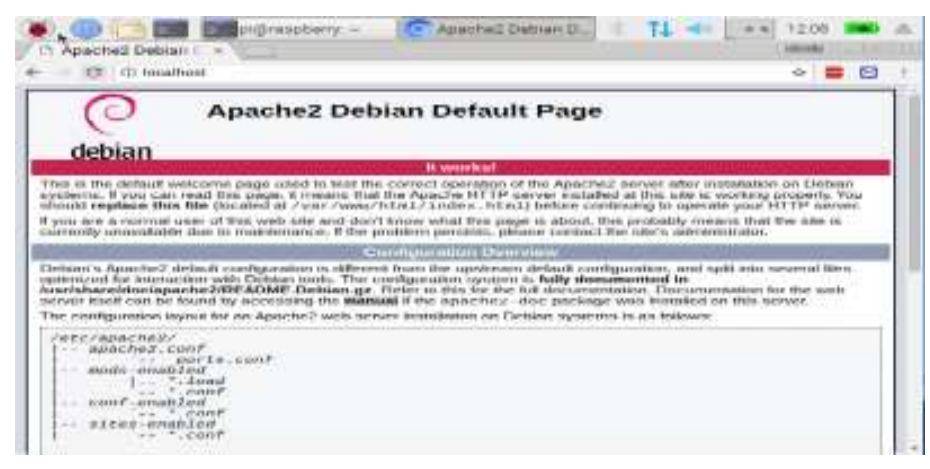

Figure 13: Apache local host.

Connect between apache and PhpmyAdmin. Write your code to control your raspberry pi according to the GPIO pins, all the input and output pins are located along the sides of the pi. It is now possible to access 40 GPIO pins while retaining the same configuration in figure 14 . 


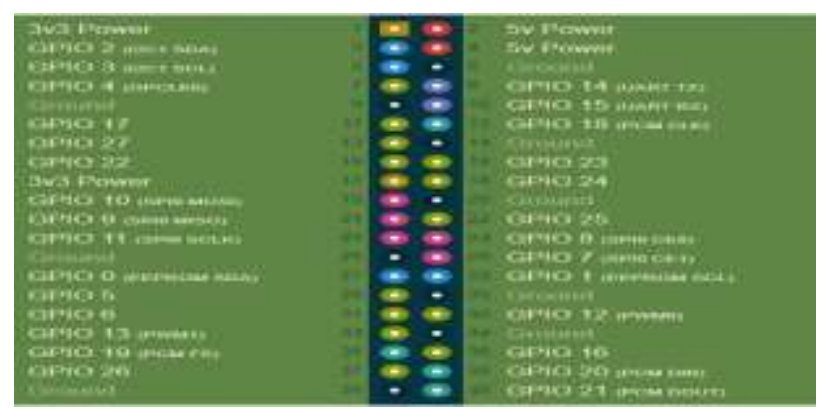

Figure 14: Raspberry pi GPIO.

Run your apache server; make your program auto-start when your raspberry pi is powered on. Install Firebase, create your cloud database project, add your firebase project credentials in the program which controls your CNC, and detect the alarm. Finally, install Flask API to control the system remotely.

\subsection{Circuit and Hardwar Connections}

Before connecting to the $\mathrm{CNC}$ machine we need to set I/O connectors $\mathrm{CN} 1$ which is responsible for digital input/output and pulses coming from the controller like figure 15 to get the alarm and the reset signals, using the alarm signal to start our diagnosis cycle and hold the running G-CODE, using the reset signal as an input to restart the drivers. I used ASDA-A2 series servo motor drivers manual user.

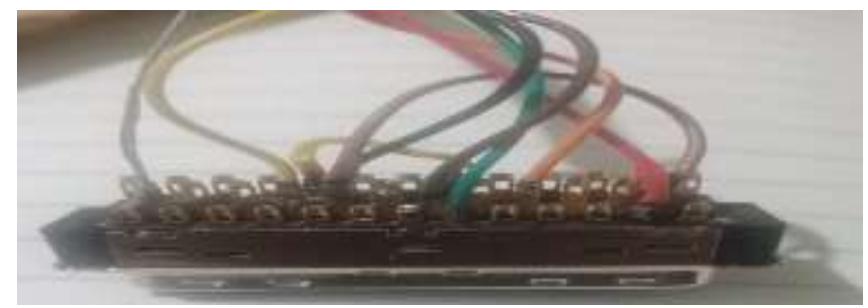

Figure 15: driver I/O connector.

You need a relay circuit and a contactor to send and receive signals coming from CNC to raspberry pi and vise verse see figure 16.

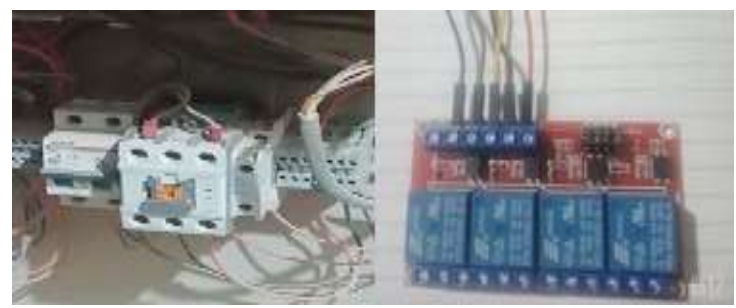

Figure 16: Contactor and relay.

Connect your raspberry pi, webcam; relay circuit, and I/O wiring figure 17 and keep an eye on your machine, you can control it locally or remotely. There is not any loss in material, time, effort, and no constant need for spare parts periodically. 


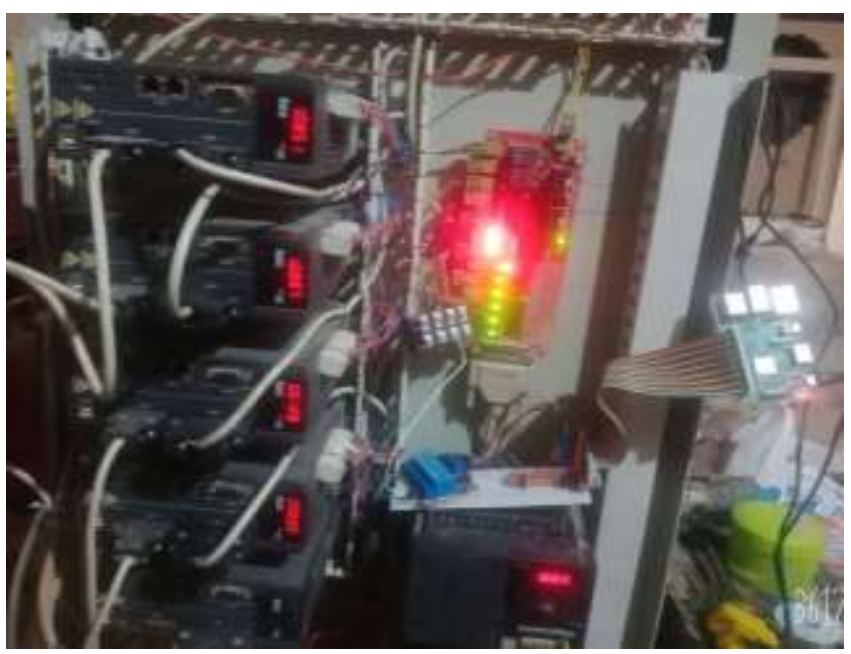

Figure 17: Faults diagnose circuit.

If an alarm has occurred, the machine pauses automatically with no movement and the GCode running program stopped at the same time as the alarm. You will get a message on your mail, that there is a malfunction in the machine, with a picture of the alarm code attached and simply you get the alarm diagnostic message figure 18 for instance Alarm 009 it occurs when the machine makes a deviation of the position.

\begin{tabular}{|c|c|c|}
\hline Causes & Checking Method & Corrective Actions \\
\hline $\begin{array}{l}\text { Parameter P2-35 is set } \\
\text { too small }\end{array}$ & $\begin{array}{l}\text { Check the setting value of } \\
\text { parameter P2-35 (The warning } \\
\text { condition of excessive position } \\
\text { deviation) }\end{array}$ & $\begin{array}{l}\text { Increase the setting value of P2-35 } \\
\text { (The warning condition of } \\
\text { excessive position deviation) }\end{array}$ \\
\hline $\begin{array}{l}\text { The setting of the gain } \\
\text { value is too small. }\end{array}$ & $\begin{array}{l}\text { Check if the setting value is } \\
\text { appropriate }\end{array}$ & Correctly adjust the gain value \\
\hline $\begin{array}{l}\text { The torque limit is too } \\
\text { low. }\end{array}$ & Check the torque limit value & $\begin{array}{l}\text { Correctly adjust the torque limit } \\
\text { value }\end{array}$ \\
\hline Excessive external load & Check the externaiload & $\begin{array}{l}\text { Reduce the external load or } \\
\text { evaluate the motor capacity again }\end{array}$ \\
\hline $\begin{array}{l}\text { Improper setting of E. } \\
\text { gear ratio }\end{array}$ & $\begin{array}{l}\text { Make sure if the proportion of P1-44 } \\
\text { and P } 1-45 \text { is appropriate. }\end{array}$ & Correctly setup E-gear ratio \\
\hline
\end{tabular}

Figure18: Diagnostic message.

Open your control page can control your machine from a computer or smartphone figure 19 from your page you can turn on/off the machine or restart all servo drivers.

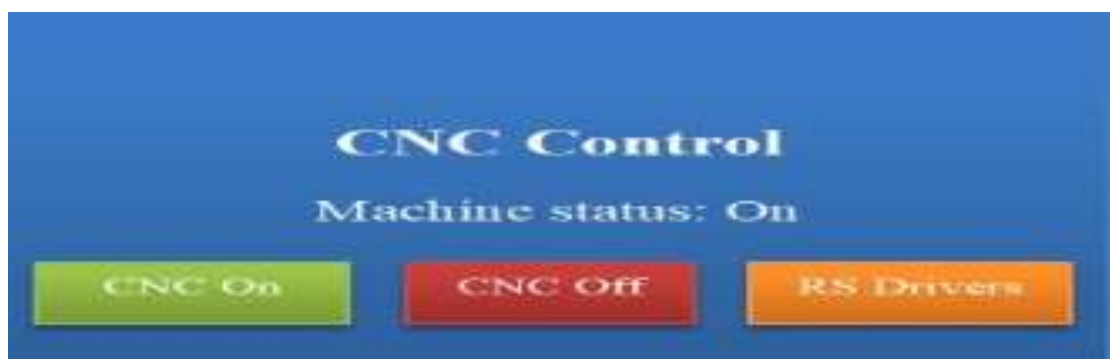

Figure 19: Control page. 


\section{CONCLUSION}

The purpose of this project is to improve communication between machines and humans, by leveraging wireless technology and machine learning algorithms, and other technological advances. We succeed to build a fault diagnostic CNC system by monitoring the warning panel of CNC drivers using a webcam, raspberry pi 3, and optical character recognition (OCR). We used raspberry pi as a sensor to indicate the errors coming from servo parameters for the absolute system. The webcam immediately cached the warning signal coming from the $\mathrm{CNC}$ to the raspberry pi and both $\mathrm{CNC}$ system engines and the G-code stopped running. At the same time, the user received a message sent by raspberry pi via e-mail that there is a fault in the system, with an image of the alarm code attached. He controlled the CNC machine remotely and gets the alarm diagnostic message from the local host which contains a database of alarm codes and a remote CNC control page. Firebase cloud recorded an alarm and sent a notification to maintenance officials. The proposed system achieved efficiency, accuracy, and speed of maintenance. It is cheap and easily upgradable.

\section{FUTURE WORK}

By adding different types of sensors along with the proposed system we can control more CNC machine functions. We can apply this fault diagnosis technique to many different systems with the change of database. Interfacing with servo motor can be useful further to automatically fault correction. Smart things compatible alarm system is a potential upgrade of the proposed system, which notifies intrusion by a loud alarm. Programmers and operators can no longer undergo trial and error on the actual parts only like Parts and machines, but now these processes can be carried out virtually in increasingly powerful software. As part of the Industry 4.0 initiative, businesses are introducing new layers of control, automation, and data collection over their machines and processes. The ever-increasing volume of data available to businesses, combined with increased capacity to analyze and interpret these data, will provide new insights into their machining processes and the general business practices that were previously unsolicited. In short, all CNC machine developers are seeking to apply artificial intelligence, so we will see a smarter and faster version of it.

\section{ACKNOWLEDGEMENT}

We would like to express our gratitude to our department professors, who guided us throughout this research paper. We take this opportunity to express our deep sense of gratitude for their invaluable guidance, ongoing encouragement, enormous motivation, which has sustained our efforts at all, the stages of research development. We would also like to thank our sponsor (Smart House Furniture Company), colleagues, and our families who supported us.

\section{REFERENCES}

[1] Georgi M. Martinov, Aleksandr B. Ljubimov, Lilija I. Martinova From classic CNC systems to cloud-based technology and back, Robotics and Computer-Integrated $\begin{array}{llll}\text { Manufacturing, } & \text { Volume } & 63,2020, & \text { ISSN }\end{array}$ https://doi.org/10.1016/j.rcim.2019.101927.

[2] L.E.S. Oliveira, A.J. Álvares. Axiomatic Design Applied to the Development of a System for Monitoring and Teleoperation of a CNC Machine through the Internet, Procedia CIRP

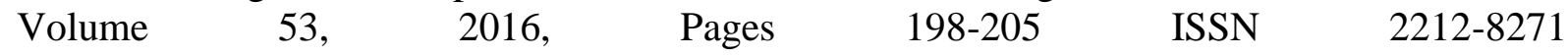
https://doi.org/10.1016/j.procir.2016.06.099. 
[3] Xu X. From cloud computing to cloud manufacturing. Robotics and Computer-Integrated Manufacturing2012; 28:75-86.orum: Cologny, Switzerland, 2016.

[4] Li B-H, Zhang L, Wang S-L, Tao F, Cao J, Jiang X, Song X, and Chai X. Cloud manufacturing: a new service-oriented networked manufacturing model. Computer Integrated Manufacturing Systems 2010; 16:1-7.

[5] Isermann, R. (2011). Fault-Diagnosis Applications: Model-Based Condition Monitoring: Actuators, Drives, Machinery, Plants, Sensors, and Fault-tolerant Systems.

[6] Felix C. Gomez de Leon Hijes Maintenance strategy based on a multi-criterion classification of equipment, Reliability Engineering \& System Safety, 2006,91(4) pp. 444-451

[7] SU Qian, ZHANG Qunsheng, Security Control Design For Computer Numerical Control Machine Tool Based On PLC, Machine Tool \& Hydraulics, 2012, 40(11) pp. 169171.

[8] Electrical control design of CNC equipment fault based on part quality Yi He1, Xuezhen Chen1, Guobo Zhao1, and Ying Li1 Published under licence by IOP Publishing Ltd, 2019 draulics, 2012, 40(11) pp. 169-171.

[9] He X (2016) recent development in reliability analysis of NC machine tools. Int J Adv Manuf Technol 85(1-4):115-131. https://doi. org/10.1007/s00170-015-7926-0.

[10] Goyal D, Pabla BS (2015) Condition-based maintenance of machine tools - a review. CIRP J Manuf Sci Technol 10:24-35.

[11] Siddhpura A, Paurobally R (2013) A review of flank wear prediction methods for tool condition monitoring in a turning process. Int J Adv Manuf Technol 65:371-393.

[12] Li, D. H., Nie, H. F., \& Peng, M. (2016). Fault diagnosis and analysis of motorized spindle Equipment Manufacturing Technology, 05, 189-190.

[13] Qiang Zhou,Ping Yan,Yang Xin, Research on a knowledge modelling methodology for fault diagnosis of machine tools based on formal semantics,2017.

[14] Alex Patricio Toapanta Guacapiña. Machine Monitoring Based on Cyber-physical Systems for Industry 4.0, November 2020. Robot. Comput. Integr. Manuf.

https://www.researchgate.net/publication/346107347

[15] Google, Google App Engine, 2020. < https://cloud.google.com/appengine/ > (Accessed October 15, 2020).

[16] Mano, L.Y.; Faiçal, B.S.; Nakamura, L.H.V.; Gomes, P.H.; Libralon, G.L.; Meneguete, R.I.; Filho, G.P.R.;

Giancristofaro, G.T.; Pessin, G.; Ueyama, J.; et al. Exploiting IoT technologies for enhancing Health Smart Homes through patient

identification and emotion recognition. Comput. Commun. 2016, 89, 178-190.

[17] Borges PV, K.; Izquierdo, E. A probabilistic approach for vision-based fire detection in videos. IEEE Trans.

Circuits Syst. Video Technol. 2010, 20, 721-731.

[18] Smith, R. An overview of the tesseract OCR engine. In Proceedings of the Ninth International Conference on

Document Analysis and Recognition (ICDAR 2007), Parana, Brazil, 23-26 September 2007. 
[19] Kim, H.; Jung, W.-K.; Choi, I.-G.; Ahn, S.-H. A Low-Cost Vision-Based Monitoring of Computer Numerical Control (CNC) Machine Tools for Small and Medium-Sized Enterprises (SMEs). Sensors 2019, 19, 4506.

[20] Ryu, M.; Kim, J.; Yun, J. Integrated semantics service platform for the Internet of Things: A case study of a smart office. Sensors 2015, 15, 2137-2160.

[21] Yun, J.; Ahn, I.-Y.; Choi, S.-C.; Kim, J. TTEO (Things Talk to Each Other): Programming smart spaces based on IoT systems. Sensors 2016, 16, 467.

[22] Bonomi, F.; Milito, R.; Zhu, J.; Addepalli, S. Fog computing and its role in the internet of things. In Proceedings, 2012.

[23] MQTT V3.1 Protocol Specification, International Business Machines Corporation (IBM) Eurotech.

[24] Z. Shelby, RFC-7959 Block-Wise Transfers in the Constrained Application Protocol (CoAP), Aug 2016.

[25] K. Fysarakis, I. Askoxylakis, O. Soultatos, I. Papaefstathiou, "Which IoT Protocol? Comparing

standardized approaches over a common M2M application,” 2016 IEEE Global Communications Conference (GLOBECOM), Washington, DC, 2016, pp. 1-7.

[26] H. Eslava, L. A. Rojas, R. Pereira, "Implementation of Machine-to Machine Solutions Using MQTT Protocol in Internet of Things (IoT) Environment to Improve Automation Process for Electrical Distribution Substations in Colombia, Journal of Power and Energy Engineering, 2015, 3, 92-96.

[27] H. Kamutzki, "Using MQTT In Real-World M2M Communication," Talk on MicroDoc blog.

[28] J. Butts, "The Information Revolution for the Enterprise," IBM. https://opcfoundation.org/information-revolution-2014/

[29] Nazir, S., \& Kaleem, M. (2019).Reliable image notifications for smart home security with MQTT.In 2019 International Conference on Information Science and Communication Technology, ICISCT 2019 IEEE. https://doi.org/10.1109/CISCT.2019.8777403. 